\title{
REATOR ELETRÔNICO AUTO-OSCILANTE "VALLEY-FILL" COM CORREÇÃO DO FATOR DE CRISTA UTILIZANDO MODULAÇÃO EM FREQÜÊNCIA
}

\author{
Álysson R. Seidel \\ arseidel@upf.br \\ Douglas Pappis \\ douglasp@mail.ufsm.br
}

\author{
Marco A. Dalla Costa \\ marcodceieee.org
}

Ricardo N. do Prado

rnpradodieee.org

GEDRE - PPGEE - UFSM

CEP 97105-900 - Santa Maria - RS

\begin{abstract}
This paper presents a self-oscillating electronic ballast employing a modified valley-fill filter in order to meet IEC 61000-3-2 Class C requirements. In order to overcome the drawback of high fluorescent lamp current crest factor, which is inherent to the valley fill filter, it is employed a frequency modulation system that keeps the crest factor lower than 1.7 limit using a simple and low cost circuit. This system uses the resonant filter features added to the possibility to control self-oscillating frequency using electronic ballast bus voltage feature. Simulation and experimental results are presented to demonstrate the system performance.
\end{abstract}

KEYWORDS: electronic ballast, power electronics, powerfactor, fluorescent lamp, self-oscillating, valley-fill filter, fluorescent lamp.

\section{RESUMO}

Neste artigo, apresenta-se um reator eletrônico autooscilante empregando o filtro valley-fill modificado como método de correção do fator de potência, de modo a atender a norma IEC 61000-3-2 Classe C. Para solucionar o problema do alto fator de crista da corrente na lâmpada fluorescente inerente ao filtro "valley-fill", emprega-se um

Artigo Submetido em 10/05/04

1a. Revisão em 15/05/04;

2a. Revisão em 09/05/05;

Aceito sob recomendação do Editor Associado

Prof. Dr. José Antenor Pomilio sistema de modulação em freqüência. Um circuito simples e de baixo custo mantém o fator de crista abaixo do limite de 1,7. São utilizadas as características de ganho do filtro ressonante aliadas à possibilidade de controlar a freqüência do circuito de comando auto-oscilante a partir da característica da tensão de barramento do reator.

PALAVRAS-CHAVE: auto-oscilante, eletrônica de potência, fator de potência, reator eletrônico, lâmpada fluorescente e valley-fill.

\section{INTRODUÇÃO}

Comparados a reatores eletromagnéticos para lâmpadas fluorescentes, reatores eletrônicos são uma realidade, tornando-se atrativos no mercado devido aos seus conhecidos méritos, comparados aeletromagnéticos, tais como: maior rendimento luminoso $(\mathrm{lm} / \mathrm{W})$, ausência de cintilamento (flicker) e ruído audível, menores peso e volume.

Reatores eletrônicos sem correção de fator de potência (FP) utilizam um capacitor de barramento de alto valor para filtrar a tensão retificada da fonte de alimentação, apresentando um bom resultado de fator de crista (FC) da corrente na lâmpada $(\mathrm{FC}<1,7)$. No entanto, apresentam baixo fator de potência $(\mathrm{FP}<0,6)$ e alta taxa de distorção harmônica (TDH $>130 \%)$. Para superar essas desvantagens, são usados dois métodos de correção do FP: 1) ativos (Verderber et al., 1994; Youn et al, 1997; Yang e Chen, 1996) e 2) passivos: (Spangler et al., 1996; Kheraluwala et al., 1995; Konopka et al., 1999; e Sum, 2000). O filtro "valley fill" (VF) é um método passivo, podendo ser usado 
para atender os limites estabelecidos pela norma IEC 61000-3-2 Classe C (Sum, 1997 e 2000). Assim, o filtro VF possui a vantagem de ser uma estrutura simples que não apresenta esforços de tensão e de corrente nos interruptores. Porém, um alto $\mathrm{FC}$ da corrente na lâmpada é inerente ao funcionamento do mesmo. Na literatura, são apresentadas soluções utilizando modulação em freqüência associada a característica de ganho do filtro ressonante de saída (Song et al., 2001). No entanto, o autor não apresenta o circuito utilizado para tal, apresentando apenas o diagrama de blocos do mesmo. Deste modo, não é possível avaliar a complexidade e o custo do circuito utilizado. Da mesma forma, as formas de onda de tensão e de corrente de entrada são omitidas, o que impossibilita avaliar a influência de cada harmônica em relação a TDH da corrente de entrada.

Entre os comandos existentes, o comando auto-oscilante possui diversas qualidades, tais como: simplicidade, confiabilidade e baixo custo. Além disso, existe a possibilidade de controle da freqüência desse sistema conforme é demonstrado por Seidel et al. (2003).

Um sistema que associe as vantagens do filtro passivo VF e do comando auto-oscilante é possível, evitando utilizar circuitos complexos e componentes adicionais, para manter o FC da corrente na lâmpada dentro do limite permitido e atender à norma IEC 61000-3-2 relativa à corrente de entrada. Neste trabalho propõe-se: 1) utilizar o filtro VF modificado para corrigir a TDH da corrente de entrada e garantir alto fator de potência; 2) utilizar o circuito de comando auto-oscilante com modulação em freqüência para corrigir o alto FC da corrente da lâmpada. Assim, obtém-se um circuito simples e de baixo custo através do uso adequado do circuito de comando auto-oscilante, o que viabiliza aplicações de baixa potência com correção de FP.

O trabalho é organizado da seguinte maneira: a seção 2 apresenta uma discussão sobre as técnicas para correção do FP existentes; na seção 3 , é apresentado o princípio utilizado para correção do FC da corrente da lâmpada; a seção 4 mostra o procedimento de projeto para o reator proposto; a seção 5 apresenta resultados experimentais. A seção 6 mostra uma discussão e a conclusão a respeito do trabalho desenvolvido.

\section{CIRCUITOS PARA CORREÇÃO DO FP}

Existem dois métodos básicos para correção do FP.

\subsection{Métodos Ativos Para Correção do FP}

Os métodos ativos para correção do FP são limitados pelo seu alto custo e complexidade. O compartilhamento de um dos interruptores é uma boa alternativa para resolver essas desvantagens. No entanto, isso geralmente resulta em esforços de tensão e/ou corrente nos interruptores controlados do circuito.

\subsection{Métodos Passivos Para Correção do FP}

Os métodos passivos para correção do FP possuem algumas vantagens em relação aos ativos, tais como: baixo custo, simplicidade e ausência de esforços de tensão e de corrente. Um dos mais simples e conhecidos métodos passivos é o filtro VF convencional, o qual é mostrado na Figura 1 e é descrito em detalhes nos itens a seguir.

A desvantagem de utilizar esse método é que a tensão retificada da fonte de alimentação tem uma filtragem parcial, o que resulta em uma ondulação na tensão de barramento $\left(\mathrm{V}_{\mathrm{DC}}\right)$ de cerca de $50 \%$, resultando um alto $\mathrm{FC}$ da corrente da lâmpada. O filtro VF básico e suas etapas de operação são mostrados na Figura 1. As etapas de operação apresentadas a seguir representam um semi-ciclo da tensão da fonte de alimentação. O outro semi-ciclo é idêntico, não necessitando ser representado.

Etapa 1) Nesta etapa, o valor instantâneo da tensão da fonte de alimentação está em um valor menor que $\mathrm{V}_{\mathrm{P}} / 2, \mathrm{D}_{5}$ e $\mathrm{D}_{7}$ estão diretamente polarizados e os demais diodos não. Neste caso, os capacitores de filtro $\left(\mathrm{C}_{\mathrm{f} 1}\right.$ e $\left.\mathrm{C}_{\mathrm{f} 2}\right)$, em paralelo, estarão fornecendo energia à carga, decaindo assim seu valor de tensão de um valor $\Delta \mathrm{V}$. Nota-se que, nesta etapa, a fonte de alimentação não está fornecendo corrente ao circuito.

Etapa 2) Assim que o valor instantâneo da tensão da fonte de alimentação ultrapassar o valor de $\mathrm{V}_{\mathrm{P}} / 2-\Delta \mathrm{V}$, a fonte de alimentação passa a alimentar a carga diretamente, pois $\mathrm{D}_{1}$ e $\mathrm{D}_{4}$ (além de $\mathrm{D}_{2}$ e $\mathrm{D}_{3}$ no próximo semi-ciclo da tensão da fonte de alimentação) estarão diretamente polarizados e os demais diodos não. Nesta etapa a tensão de barramento terá a ondulação da tensão da fonte de alimentação.

Etapa 3) $\mathrm{O}$ diodo $\mathrm{D}_{6}$ começa a conduzir quando a tensão da fonte de alimentação atingir o valor de $\mathrm{V}_{\mathrm{P}}-2 \Delta \mathrm{V}$. A fonte de alimentação, além de fornecer energia à carga, também carregará os capacitores eletrolíticos em série, o que exigirá um valor de corrente maior nesta etapa. A tensão de barramento permanece igual à tensão da fonte de alimentação.

Etapa 4) Quando a tensão da fonte de alimentação atingir seu valor de pico $\left(V_{P}\right)$ os capacitores de filtro $\left(C_{f 1}\right.$ e $\left.C_{f 2}\right)$ carregar-se-ão com o valor de $\mathrm{V}_{\mathrm{P}} / 2$, cada um, e esta etapa terá seu início. Quanto ao funcionamento, esta é idêntica à etapa 2. 
As formas de onda teóricas podem ser observadas na Figura 2 , onde é mostrada a tensão de entrada do reator $\left(\mathrm{V}_{\text {ent }}\right)$, corrente de entrada $\left(\mathrm{I}_{\text {ent }}\right)$ e tensão de barramento $\left(\mathrm{V}_{\mathrm{DC}}\right)$.
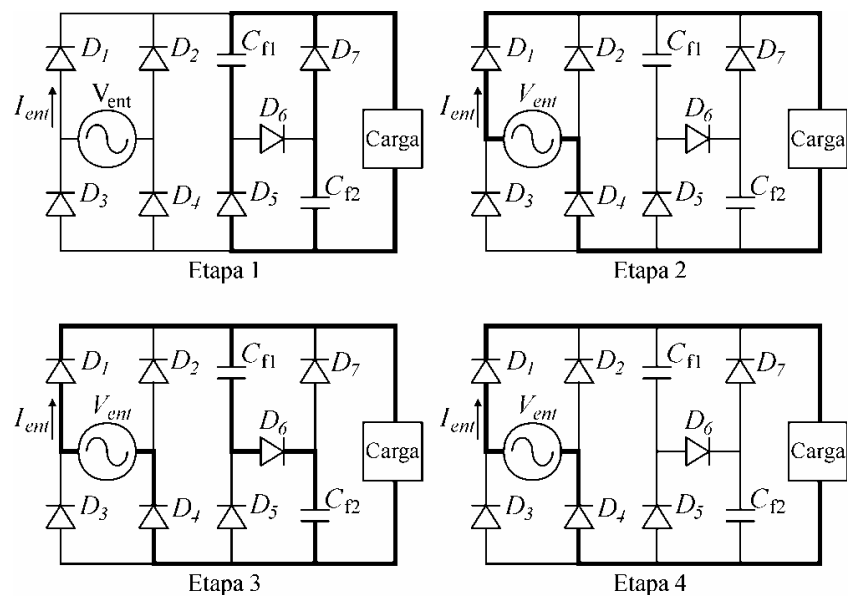

Figura 1. Etapas de operação do filtro VF convencional.

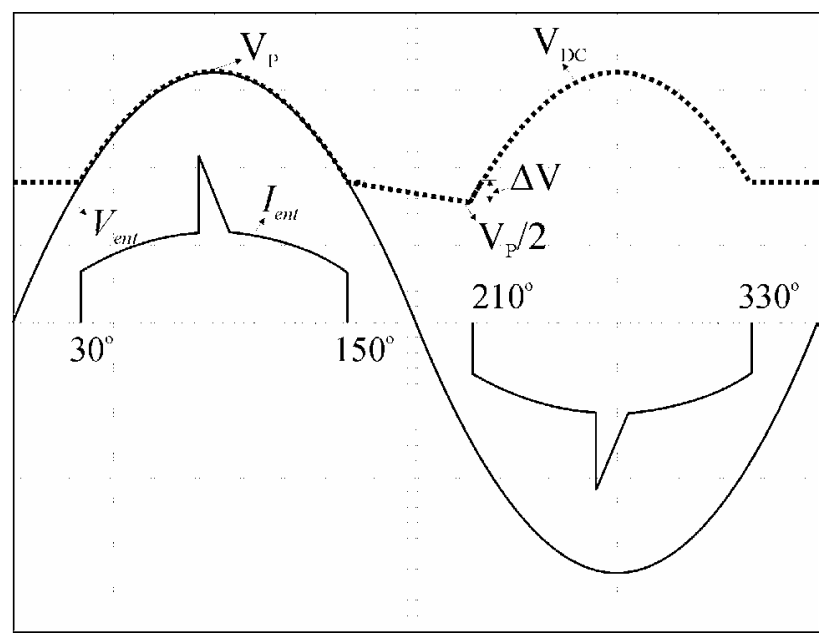

Figura 2. Formas de onda teóricas do filtro VF. associa a utilização de um transistor em uma configuração bidirecional, como proposto em Michel et al. (2002).

Nota-se que, apesar de aumentar o tempo de condução da corrente da fonte de alimentação, a corrente de entrada ainda se encontra fora da norma IEC 61000-3-2. Deste modo, para adaptar o filtro VF à norma, algumas estruturas tem sido apresentadas na literatura (Spangler et al., 1991, e Kheraluwala et al., 1995).

Entre as alternativas de modificação do filtro "valley-fill", há: algumas que não atendem à norma IEC 61000-3-2 (Marques et al., 2000); uma alternativa que aplica uma parcela de corrente contínua na lâmpada como decorrência da modificação do filtro VF (Konopka et al., 1999); alternativas que corrigem o problema do alto fator de crista, mas, por conseqüência, deterioram a corrente de entrada (Youn et al., 1997; Konopka et al., 1999; Marques et al., 2002); alternativas que corrigem o problema da distorção da corrente de entrada, mas apresentam um aumento significativo da ondulação da tensão do barramento (Kheraluwala et al., 1995, Konopka et al., 1999; Sum, 2000). Uma alternativa que corrige o problema da distorção harmônica da corrente de entrada sem um acréscimo significativo da ondulação da tensão de barramento é apresentada em Sum (1997).

Apesar de alguns desses circuitos atenderem à norma que limita a TDH da corrente de entrada, algumas configurações apresentam alto $\mathrm{FC}$ da corrente na lâmpada $(\mathrm{FC}>2,1)$, devido à ondulação da tensão de barramento $\left(\mathrm{V}_{\mathrm{DC}}\right)$.

Deste modo, uma boa alternativa é a utilização do filtro VF modificado com modulação em freqüência, de modo a atender às exigências da entrada (linha) e saída (lâmpada).

A técnica de controle utilizada neste trabalho é baseada na modulação em freqüência proposta em Seidel et al. (2003), a qual tem como base a inserção de elementos passivos em paralelo ao circuito de comando auto-oscilante.

\section{PRINCÍPIO DE FUNCIONAMENTO DO SISTEMA PROPOSTO PARA CORREÇÃO DO FC}

$\mathrm{O}$ princípio da correção do $\mathrm{FC}$ da corrente na lâmpada baseia-se na utilização da ondulação da tensão de barramento do circuito.

A melhoria do FC pode ser obtida por meio do circuito da Figura 3a, que emprega a tensão do barramento (Figura 3b) como regra de controle (ON-OFF, basicamente). Se a tensão do barramento é maior que $\mathrm{Vp} / 2$, o transistor está em saturação (conduzindo), o que introduz Lc no circuito de disparo, diminuindo o ganho do filtro ressonante e aumentando a freqüência de operação do reator (Figura 3c). Por outro lado, para uma tensão de barramento inferior a $\mathrm{Vp} / 2$, o transistor está em corte (aberto) e a freqüência diminui (Figura 3d). Esse mecanismo atua de forma a reduzir as divergências entre os valores eficazes da corrente na lâmpada durante as etapas de funcionamento do filtro, reduzindo também o FC.

A Figura 4 mostra o sistema proposto, destacando-se o circuito de comando auto-oscilante, o filtro VF modificado e o circuito de modulação em freqüência. 


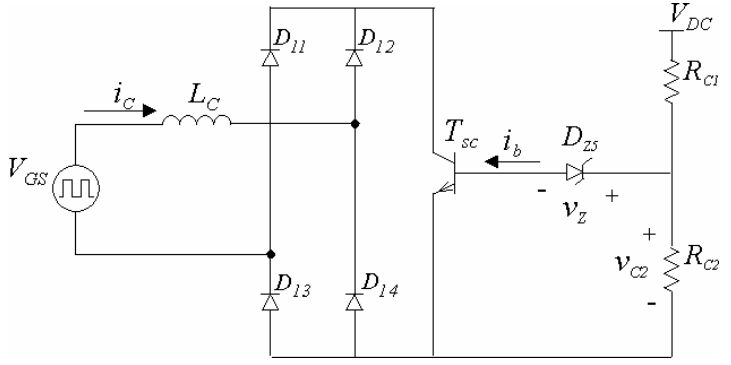

(a)

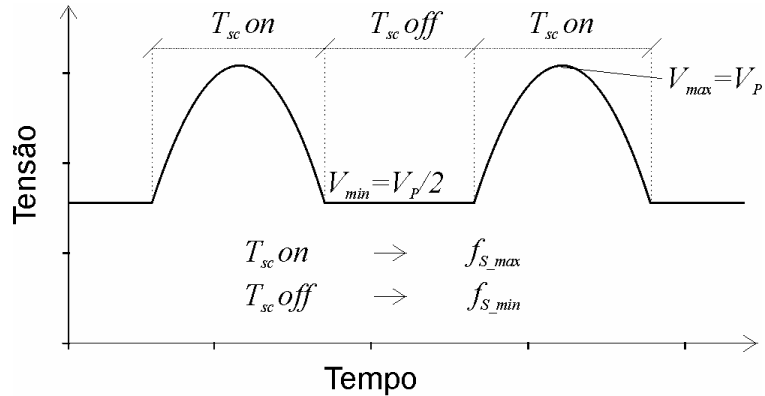

(b)

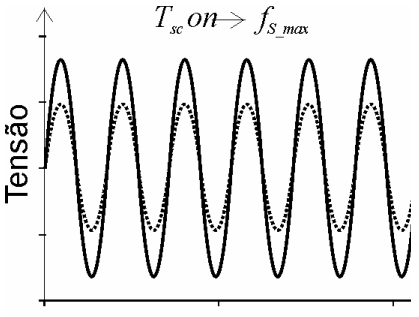

Tempo

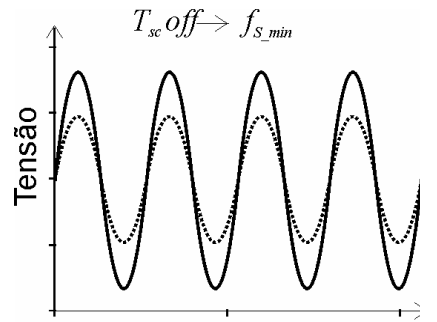

Tempo

(d)
Figura 3. Princípio de funcionamento do circuito proposto: (a) circuito simplificado; (b) tensão de barramento; (c) simulação da tensão e da corrente na lâmpada (freqüência máxima); (d) simulação da tensão e da corrente na lâmpada (freqüência mínima).

\section{PROCEDIMENTO DE PROJETO}

O projeto do sistema proposto envolve duas etapas: a primeira refere-se ao projeto do filtro ressonante (Bisogno et. al., 2001); a segunda, ao projeto do circuito de modulação da freqüência de operação, conforme Seidel et al. (2003).

\subsection{Projeto do Filtro Ressonante}

Para determinar os parâmetros do filtro LCC ressonante, algumas aproximações são consideradas:
Aproximação fundamental $V_{f}$, que consiste em considerar somente a componente fundamental da forma de onda $V_{a b}$ aplicada ao filtro ressonante;

- A lâmpada fluorescente é representada por sua resistência em regime permanente $(\mathrm{R})$ e, no caso da partida, por 100R;

- Os componentes do filtro ressonante $\left(L, C_{P}\right.$ e $\left.C_{S}\right)$ são considerados lineares e invariantes no tempo.

Os passos a seguir são usados para determinar os componentes do filtro ressonante:

\subsubsection{Dados de Entrada}

A freqüência de operação e a tensão aplicada ao filtro ressonante são determinadas, considerando a tensão de barramento resultante do filtro VF $\left(V_{\min }\right)$.

\subsubsection{Parâmetros do Filtro Ressonante}

Os parâmetros do filtro ressonante $\left(L, C_{P}\right.$ e $\left.C_{S}\right)$ são determinados a partir do seguinte:

a) Angulo de Fase $\phi$ - O ângulo de fase é determinado de modo a garantir a partida da lâmpada fluorescente, potência em regime permanente e operação com comutação suave (ZVS ). Seu valor é determinado pela equação (1).

$$
\phi=\arctan \left(\left(L \omega-\frac{1}{C_{S} \omega}-\frac{R^{2} C_{P} \omega}{\left(1+C_{P}^{2} R^{2} \omega^{2}\right)}\right) \cdot \frac{1+C_{P}^{2} R^{2} \omega^{2}}{R}\right)
$$

em que:

$\omega=2 \pi f_{s} \mathrm{e}$

$\omega \quad$ - freqüência angular;

$f_{s} \quad$ - freqüência de operação;

$R \quad$ - resistência equivalente da lâmpada.

A potência da lâmpada fluorescente, representada por sua resistência equivalente $R$ é definida por (2).

$$
P(\phi)=\frac{E^{2} \cdot 2}{\pi^{2}} \cdot \frac{\left(1+C_{P}^{2} R^{2} \omega^{2}\right) R}{R^{2}+(R \cdot \tan (\phi))^{2}}
$$

em que:

$E$ - tensão de barramento $V_{\min }$;

$P$ - potência na lâmpada.

Deste modo, o ângulo de fase $\phi$ pode ser determinado graficamente, de acordo com a Figura 5. 


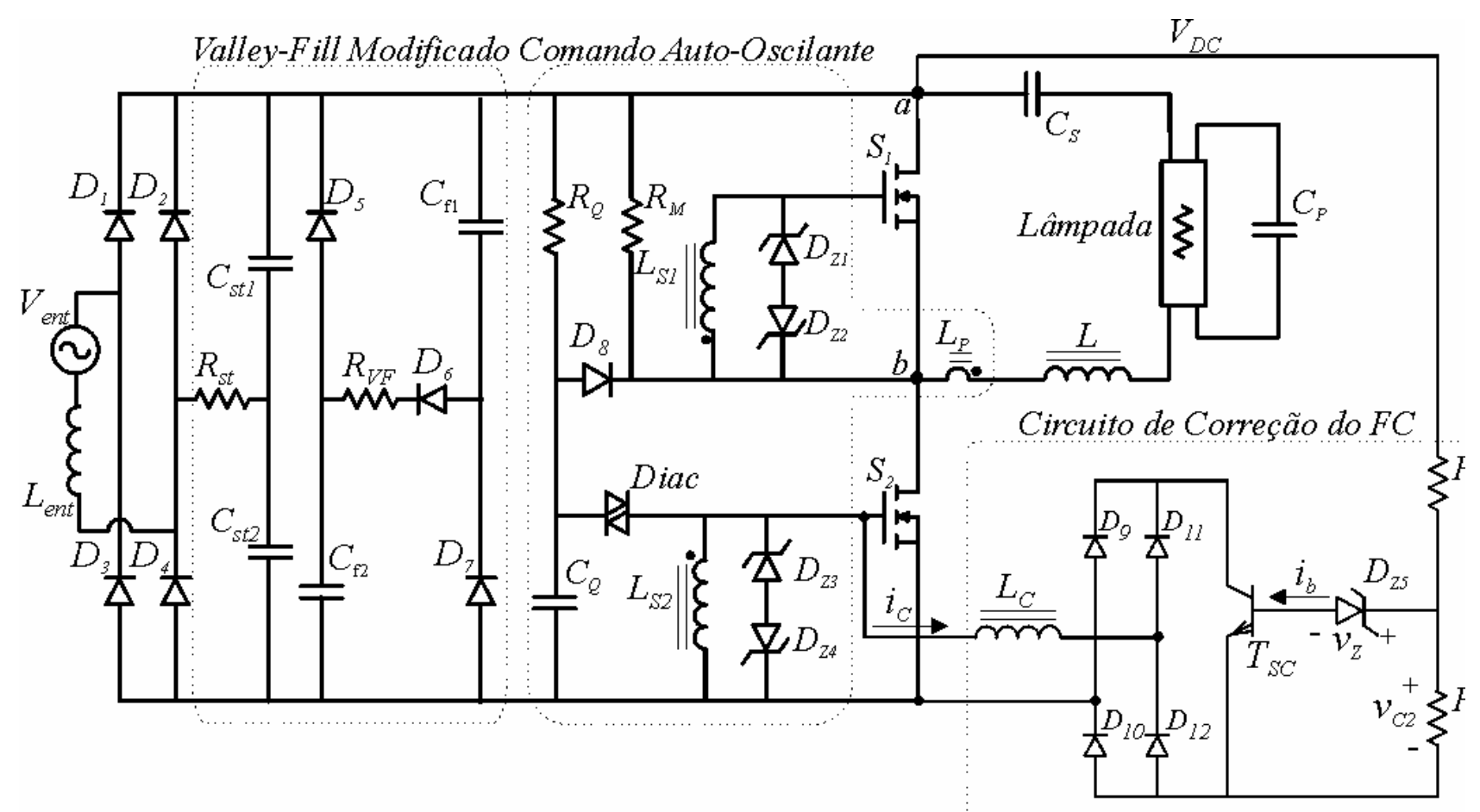

Figura 4. Circuito proposto.

b) Capacitor Paralelo $C_{p}$-A partir do ângulo $\phi$, determinado na Figura 5, determina-se o valor do capacitor paralelo por (3).

$$
C_{P}(\phi)=\frac{1}{\omega R} \sqrt{\frac{P(\phi)}{R}\left(\frac{\pi^{2}\left(R^{2}+(R \cdot \tan (\phi))^{2}\right)}{E^{2} \cdot 2}\right)-1}
$$

c) Indutor Ressonante $L-\mathrm{O}$ indutor ressonante é determinado por (4).

$$
L\left(\phi, C_{S}\right)=\frac{R \tan (\phi) \omega^{-1}+C_{P}(\phi) R^{2}}{1+\omega^{2} C_{P}^{2}(\phi) R^{2}}+\frac{1}{C_{S} \omega^{2}}
$$

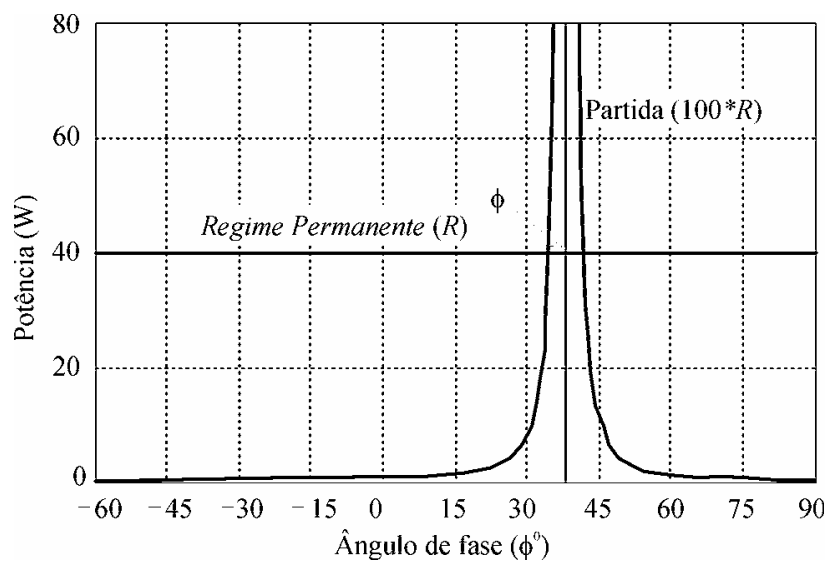

Figura 5. Potência na lâmpada versus ângulo de fase $\phi$.
Em que $C_{S}$ é um valor típico de capacitor suficiente para bloquear a componente contínua da tensão quadrada aplicada ao filtro ressonante. Assim, adota-se um valor para a capacitância $C_{S}$ de $150 \mathrm{nF}$.

Portanto, a partir de (2), (3) e (4), determina-se os componentes do filtro de saída.

\subsection{Projeto do Circuito de Correção do Fator de Crista}

Os parâmetros do circuito de comando auto-oscilante e do circuito de correção do fator de crista são determinados através das seguintes considerações:

- São considerados dois níveis para tensão $V_{a b}, V_{\min } \mathrm{e}$ $V_{\max }$

- A indutância $L c$ é determinada de modo a se obter a máxima freqüência de comutação $f_{s_{-} \max }$ para o nível máximo da tensão $V_{a b}\left(V_{\max }\right)$;

- Os parâmetros do filtro ressonante são determinados considerando a tensão mínima de barramento $V_{\text {min }}$.

\subsubsection{Comando Auto-Oscilante}

Os parâmetros do circuito de comando auto-oscilante são determinados de acordo com a metodologia de projeto apresentada em Seidel et al (2003). 


\subsubsection{Circuito de Correção do Fator de Crista}

A correção do FC da corrente na lâmpada é obtida através da variação da freqüência de operação, conforme já descrito na seção 3. A variação de freqüência é obtida pela inserção de um indutor em paralelo com o circuito de gatilho, através de um transistor que conecta o indutor $L_{c}$ quando a tensão $V_{D C}$ torna-se maior que $V_{\min }$, como pode ser visto na Figura 6.a. e 6.b. O comportamento como um sistema de controle permite que se controle o FC da corrente na lâmpada pelo controle da freqüência de operação do reator. Os parâmetros são definidos considerando a proposta mostrada em Seidel et al. (2003).



(a) Sinal de controle versus $V_{a b}$

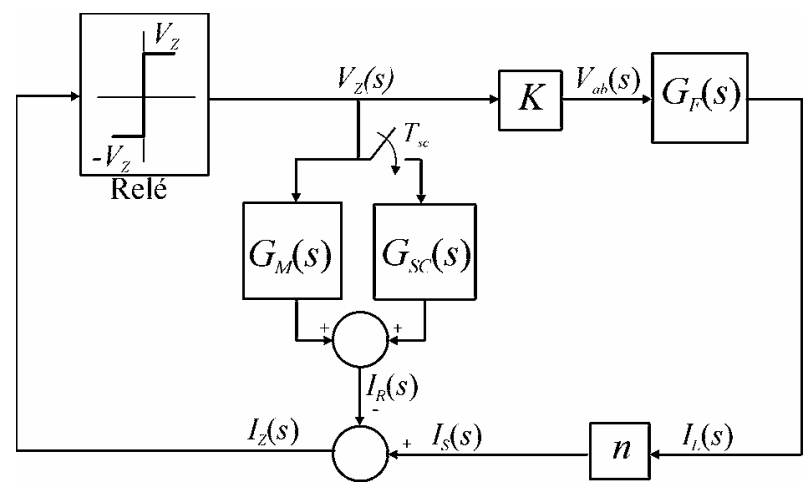

(b) Diagrama de blocos do sistema proposto.

Figura 6. Princípios do circuito de correção do FC.

\section{RESULTADOS EXPERIMENTAIS}

A Figura 4 mostra o circuito do protótipo implementado em laboratório. A Tabela I mostra a especificação dos dados de entrada, parâmetros do filtro de entrada e os valores dos principais componentes do circuito implementado.

Através da análise dos dados obtidos, o reator implementado apresentou um FP de 0,96 e THD da corrente de entrada de $15 \%$, atendendo aos limites da IEC 61000-3-2 classe C. A Figura 7 mostra a contribuição percentual de cada amplitude harmônica. O rendimento apresentado pelo reator foi de $90 \%$. Para as aquisições, foi utilizado um osciloscópio da marca Tektronix, modelo TDS 430A.
Tabela I - Principais Parâmetros

\begin{tabular}{|c|c|c|}
\hline \multicolumn{3}{|c|}{ Dados de Entrada } \\
\hline Tensão de & rada & $\begin{array}{l}V_{\text {ent }}=220 \quad V_{\text {eficaz }} \\
60 \mathrm{~Hz}\end{array}$ \\
\hline $\begin{array}{l}\text { Resistênci } \\
\text { fluorescen }\end{array}$ & divalente da lâmpada & $\mathrm{R}=204 \Omega$ \\
\hline Potência d & í́da & $40 \mathrm{~W}$ \\
\hline Faixa de fi & iências de operação & $45 \mathrm{kHz}-60 \mathrm{kHz}$ \\
\hline \multicolumn{3}{|c|}{ Reator Eletrônico Auto-Oscilante } \\
\hline$L_{p}, L_{S 1}, L_{S 2}$ & \multicolumn{2}{|c|}{$\begin{array}{l}\text { 2/10/10 voltas no núcleo T15 IP6 - } \\
\text { Thornton }\end{array}$} \\
\hline$D_{Z 1}-D_{Z 4}$ & \multicolumn{2}{|c|}{ Diodo Zener 12 V 1/2 W } \\
\hline Diac & \multicolumn{2}{|l|}{ DB3 } \\
\hline$R_{Q}$ & \multicolumn{2}{|l|}{ Resistor $220 \mathrm{k} \Omega / 1 / 8 \mathrm{~W}$} \\
\hline$C_{Q}$ & \multicolumn{2}{|c|}{ Capacitor Cerâmico 100nF / $63 \mathrm{~V}$} \\
\hline$R_{M}$ & \multicolumn{2}{|l|}{ Resistor $470 \mathrm{k} \Omega / 1 / 8 \mathrm{~W}$} \\
\hline$D_{5}$ & \multicolumn{2}{|l|}{ Diodo UF4007 } \\
\hline$S_{1}, S_{2}$ & \multicolumn{2}{|l|}{ MOSFET IRF740 } \\
\hline$D_{1}-D_{8}$ & \multicolumn{2}{|l|}{ Diodo 1N4004 } \\
\hline Lâmpada & \multicolumn{2}{|c|}{ Lâmpada OSRAM F40D-Daylight } \\
\hline \multicolumn{3}{|c|}{ Filtro Ressonante } \\
\hline$C_{S}$ & \multicolumn{2}{|c|}{ Capacitor Série Ressonante, $147 \mathrm{nF}, 250$} \\
\hline$L$ & \multicolumn{2}{|c|}{ Indutor Ressonante, $800 \mu \mathrm{H}$} \\
\hline$L_{\text {ent }}$ & \multicolumn{2}{|l|}{ Filtro de EMI $5 \mathrm{mH}$} \\
\hline \multicolumn{3}{|c|}{ Filtro VF Modificado } \\
\hline$C_{s t 1}, C_{s t 2}$ & \multicolumn{2}{|c|}{$\begin{array}{l}\text { Capacitor de Polipropileno / } 470 \mathrm{nF} 200 \\
V_{a c}\end{array}$} \\
\hline$C_{f 1}, C_{f 2}$ & \multicolumn{2}{|c|}{ Capacitor Eletrolítico / $100 \mu \mathrm{F} 250 V_{d c}$} \\
\hline$R_{V F}$ & \multicolumn{2}{|c|}{ Resistor $50 \Omega ; 1 / 2 \mathrm{~W}$} \\
\hline$R_{s t}$ & \multicolumn{2}{|l|}{ Resistor $220 \Omega ; 2 \mathrm{~W}$} \\
\hline \multicolumn{3}{|c|}{ Circuito de Modulação de Freqüência } \\
\hline$D_{9}-D_{12}$ & \multicolumn{2}{|l|}{ Diodo $4 \times 1 N 4148$} \\
\hline$R_{C 1}, R_{C 2}$ & \multicolumn{2}{|c|}{ Resistores $100 \mathrm{k} \Omega ; 10 \mathrm{k} \Omega ; 1 / 8 \mathrm{~W}$} \\
\hline$L_{C}$ & \multicolumn{2}{|c|}{ Indutor de Controle, $100 \mu \mathrm{H}$} \\
\hline$D_{Z 5}$ & \multicolumn{2}{|c|}{ Diodo Zener $12 \mathrm{~V}, 1 / 2 \mathrm{~W}$} \\
\hline$T_{S C}$ & \multicolumn{2}{|c|}{ Transistor Bipolar, 2N2222 } \\
\hline
\end{tabular}

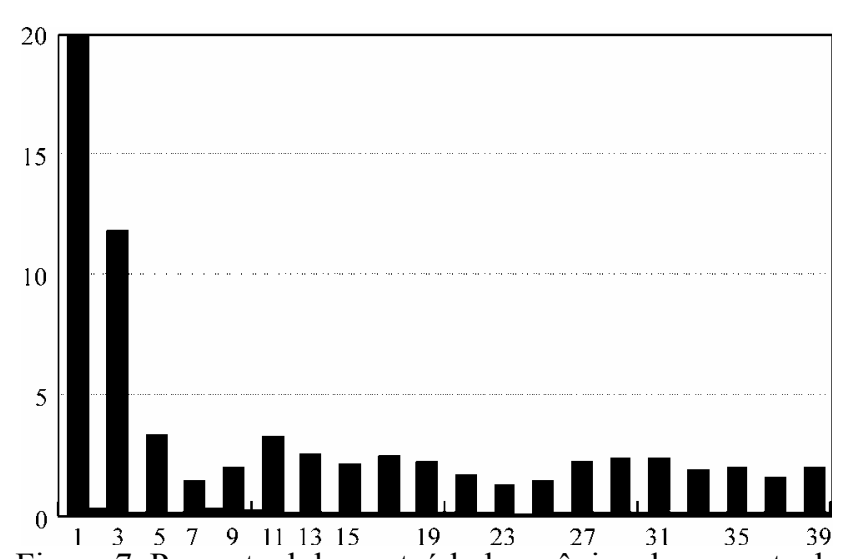

Figura 7. Percentual do conteúdo harmônico da corrente de entrada. 
A Figura 8 mostra os resultados experimentais obtidos do protótipo implementado. A Figura 8a mostra a envoltória da tensão e da corrente na lâmpada. A partir da aquisição de 40.000 pontos de amostragem, verificou-se que o FC da corrente na lâmpada é de 1,68. Nas Figuras $8 \mathrm{~b}$ e $8 \mathrm{c}$ são mostradas a tensão e a corrente na lâmpada para os casos $f_{s_{\text {min }}}(60 \mathrm{kHz})$ e $f_{s_{\text {max }}}(45 \mathrm{kHz})$, respectivamente. A Figura $8 \overline{\mathrm{d}}$ mostra a tensão de barramento $\left(\mathrm{V}_{\mathrm{DC}}\right)$ e a corrente de controle $\left(i_{c}\right)$ que é resultado da atuação do circuito de controle que permite controlar o fator de crista da corrente da lâmpada fluorescente.

A Figura 8e mostra a tensão e a corrente na entrada do reator. As Figuras $8 \mathrm{f}$ e $8 \mathrm{~g}$ mostram a comutação suave (ZVS) dos interruptores, para $f_{s_{-} \min }$ e $f_{s_{-} \max }$, respectivamente. Observa-se que a comutação suave mantém-se para toda faixa de freqüência de operação. A Figura $8 \mathrm{~h}$ mostra a tensão e a corrente na lâmpada para partida instantânea.

\section{CONCLUSÃO}

Neste trabalho, é apresentado um reator eletrônico empregando o filtro VF modificado para correção do FP. A correção do FC da corrente da lâmpada, imprescindível para sua implementação, é realizada utilizando um circuito simples e de baixo custo que efetua a modulação em freqüência descrita, a qual é realizada utilizando o circuito de comando auto-oscilante de forma apropriada.

Os resultados experimentais apresentados e sua análise demonstram a viabilidade do sistema proposto. $\mathrm{O}$ fator de crista da corrente na lâmpada menor que 1,7 , o fator de potência de 0,96 , e as contribuições de cada componente harmônica inferiores ao limite estabelecido por normas internacionais garantem os limites de saída (lâmpada fluorescente) e de entrada (fonte de alimentação).

\section{AGRADECIMENTOS}

Este projeto foi financiado pelo $\mathrm{CNPq}$ (processo 551439/01-7).

\section{REFERÊNCIAS BIBLIOGRÁFICAS}

Verderber, R. R., Morse, O. C., and Alling, W. R. (1994). Harmonics From Compact Fluorescent Lamps. IEEE Transaction on Industry Applications, vol. 29, no. 3, pp. 670-674.

Youn, Y. S., Chae, G., and Cho, G. H. (1997). A Unity Power Factor Electronic Ballast for Fluorescent Lamp Having Improved Valley-fill and Valley Boost Converter. IEEE PESC, pp. 53-59.

Yang, Y. R., and Chen, C. L. (1996). A Self-Excited HalfBridge Series-Resonant Ballast with Automatic Input Current Shaping. IEEE PESC, pp. 881-886.
Spangler, J. Hussain, B., and Behera, A. K. (1991). Electronic Ballast Using Power Factor Correction Techniques for Loads Greater than 300 Watts. IEEE APEC, pp. 393-399.

Marques, R. N., Braga, H. A. C. (2000). Valley-Fill Filters Applied to Electronic Ballasts. IEEE INDUSCON, pp. 611-616.

Kheraluwala, M. H. and El-Hamamsy, S. A. (1995). Modified Valley-Fill High Power Factor Electronic Ballast for Compact Fluorescent Lamps. IEEE PESC, vol. 1, pp. 10-14.

Sum, K. K. (1997). Improved Valley-Fill Passive Current Shaper. PowerSystems World, Baltimore, Maryland, Sept.

Konopka, J. G. (1999). Electronic Ballast With Lamp Current Valley-Fill Power Factor Correction. U.S. Patent (Nov. 30), 5,994,847.

Sum, K. K. (2000). Valley-Fill Power Factor Correction Circuit. U.S. Patent (Oct. 31), 6,141,230.

Song, J., Song, J-H., Choy, I. and Choi, J-Y., Oct. 2001. Improving Crest Factor of Electronic Ballast-Fed Fluorescent Lamp Current Using Pulse Frequency Modulation. IEEE Transaction on Industrial Electronics, vol. 48, no. 5, pp. 1015-1024.

Seidel, A. R., Bisogno, F. E, do Prado, R. N. and Pinheiro, H., Dezember 2003. Self-oscillating Dimmable Electronic Ballast. IEEE Transaction on Industrial Electronics, vol 50., no. 6.

Michel, A. L., Pappis, D., Campos, A., do Prado, R. N. (2002). Electronic Ballast with Automatic Luminous Variation and Presence Detection Using Microcontroller and Self-Oscillating Command. IEEE IAS, vol. 2, pp. 1071-1077.

Cervi, M., do Prado, R. N., Seidel, A. R., and Bisogno, F. E. (2002). Fluorescent Lamp Model Based on the Equivalent Resistance Variation. IEEE IAS, vol. 1, pp. 680-684.

Bisogno, F. E., Holsbach, R., do Prado, R. N., and Seidel, A. R. (2002), Resonant Filter Applications in Electronic Ballast. IEEE IAS, vol. 1, pp. 348-354.

Marques, R. N., e Braga, H. A. C. (2002). Valley Fill Filter Derived Electronic Ballasts - A Comparative Study. IEEE INDUSCON, vol. 2, pp. 1071-1077.

Chae, G., Youn, Y. S., and Cho, G. H. (1998). High Power Factor Correction Circuit Using Valley ChargePumping for Low Cost Electronic Ballasts. IEEE PESC, pp. 2003-2008. 




(a) $100 \mathrm{~V} / \mathrm{div} ; 0.5 \mathrm{~A} / \mathrm{div} ; 2.5 \mathrm{~ms} / \mathrm{div}$.

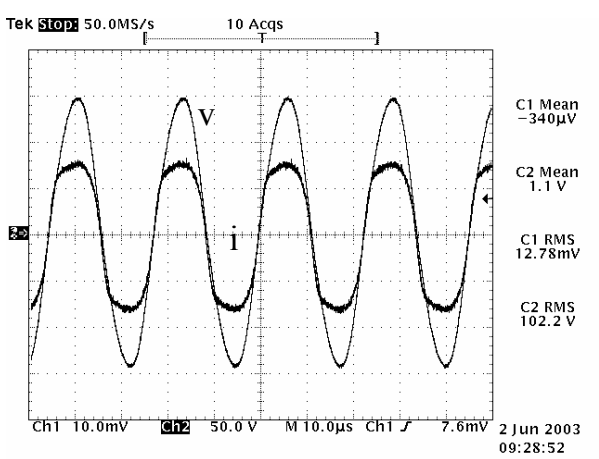

(b) $100 \mathrm{~V} / \mathrm{div} ; 0.2 \mathrm{~A} / \mathrm{div} ; 10 \mu \mathrm{s} / \mathrm{div}$.



(c) $100 \mathrm{~V} / \mathrm{div} ; 0.2 \mathrm{~A} / \mathrm{div} ; 10 \mu \mathrm{s} / \mathrm{div}$.

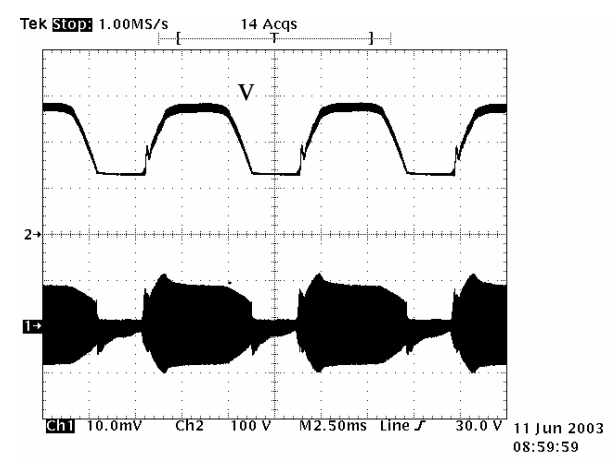

(d) $100 \mathrm{~V} / \mathrm{div} ; 0.2 \mathrm{~A} / \mathrm{div} ; 2.5 \mathrm{~ms} / \mathrm{div}$.



(e) $100 \mathrm{~V} / \mathrm{div} ; 0.5 \mathrm{~A} / \mathrm{div} ; 10 \mu \mathrm{s} / \mathrm{div}$.

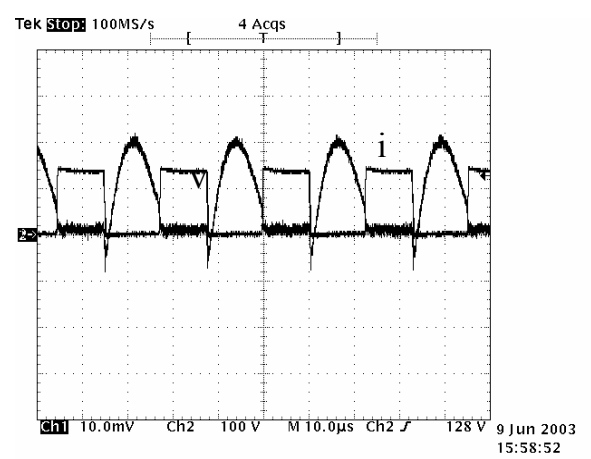

(f) $100 \mathrm{~V} / \mathrm{div} ; 0.2 \mathrm{~A} / \mathrm{div} ; 10 \mu \mathrm{s} / \mathrm{div}$.

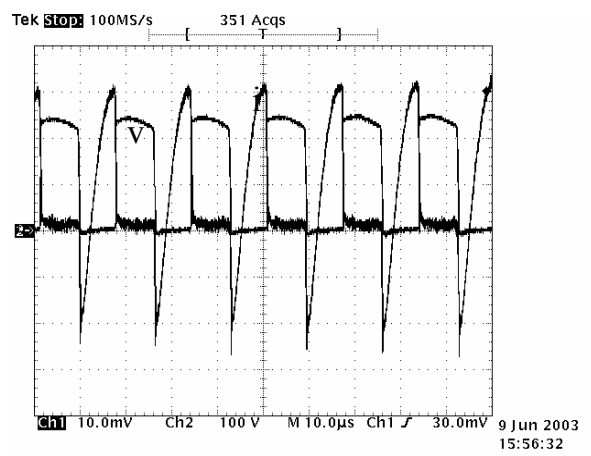

(g) $100 \mathrm{~V} / \mathrm{div} ; 0.2 \mathrm{~A} / \mathrm{div} ; 10 \mu \mathrm{s} / \mathrm{div}$.

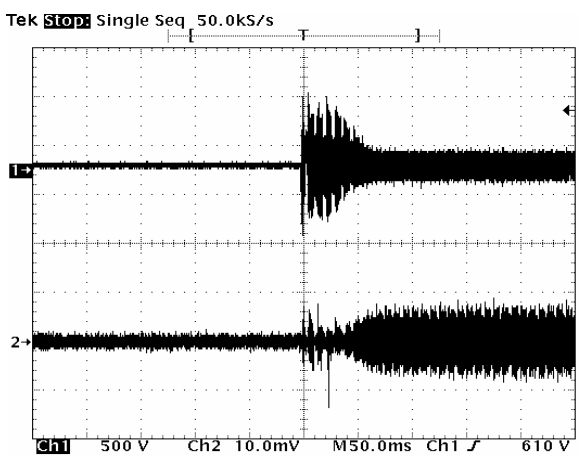

(h) $500 \mathrm{~V} / \mathrm{div} ; 1 \mathrm{~A} / \mathrm{div} ; 50 \mathrm{~ms} / \mathrm{div}$.

Figura 8. Resultados experimentais: (a) envoltória da tensão e da corrente na lâmpada; (b) tensão e corrente na lâmpada para fs $=45 \mathrm{kHz}$; (c) tensão e corrente na lâmpada para fs $=60 \mathrm{kHz}$; (d) tensão de barramento VDC e corrente de controle ic; (e) tensão e corrente de entrada; (f) tensão e corrente no interruptor S2, fs $=45 \mathrm{kHz}$; (g) tensão e corrente no interruptor $\mathrm{S} 2, \mathrm{fS}=60 \mathrm{kHz}(\mathrm{h})$ tensão e corrente na ignição da lâmpada. 